\title{
Clinical limitations of the estimation of glomerular filtration rate from height/plasma creatinine ratio: a comparison with simultaneous ${ }^{51} \mathrm{Cr}$ edetic acid slope clearance
}

\author{
J G DAVIES, C M TAYLOR, R H R WHITE, AND T MARSHALL
}

Department of Nephrology, Children's Hospital, Ladywood, Birmingham, and Department of Social Medicine, University of Birmingham

SUMMARY A retrospective comparison of single determinations of glomerular filtration rate measured by ${ }^{51}$ chromium-edetic acid slope clearance $\left(\mathrm{C}_{\text {EDTA }}\right)$ and height/plasma creatinine ratio $\left(\mathrm{Ht} / \mathrm{P}_{\mathrm{C}}\right)$ was undertaken in 199 children aged between 3 and 16 years. Analysis of the data indicated that only if $\mathrm{Ht} / \mathrm{P}_{\mathrm{C}} \leqslant 1 \cdot 2 \mathrm{~cm} / \mu \mathrm{mol}$ per 1 was the relationship between $\mathrm{Ht} / \mathrm{P}_{\mathrm{C}}$ and $\mathrm{C}_{\mathrm{EDTA}}$ linear. Over this range where renal function is significantly impaired the confidence limits for individual prediction of glomerular filtration rate were so wide that a definitive measurement of renal function would be necessary in clinical practice. Where $\mathrm{Ht} / \mathrm{P}_{\mathrm{C}}>1 \cdot 2$ the relationship was non-linear and the predictive confidence limits very wide. To apply the technique as a screening test for normal renal function $\left(C_{\text {EDTA }} \geqslant 80 \mathrm{ml} / \mathrm{min}\right.$ per $\left.1 \cdot 73 \mathrm{~m}^{2}\right)$ the $\mathrm{Ht} / \mathrm{P}_{\mathrm{C}}$ would need to exceed $2 \cdot 16$. In our study this would have detected 57 out of 131 patients who had normal glomerular filtration rates and erroneously included 2 out of 68 with subnormal renal function.

A simple and accurate test of glomerular filtration rate (GFR) would considerably expedite the investigation of children with renal disease. Creatinine clearance techniques rely on complete, precisely timed urine collections which are inherently inaccurate in patients with obstructive uropathy or reflux. ${ }^{1}$ Inulin clearance, requiring a continuous intravenous infusion, poses ethical and practical problems and few laboratories routinely measure inulin concentrations. Radioisotope slope clearance studies have been shown to be accurate except in the oedematous patient. ${ }^{2}$ They are simple to perform, but require repeated venepunctures and take at least 3 hours, rendering them unsuitable for routine outpatient work.

In a growing child the level of plasma creatinine $\left(P_{C}\right)$ varies inversely with GFR but the relationship is not a simple one and, although GFR per surface area is constant above 3 years of age ${ }^{3}$ endogenous creatinine production and mean $P_{C}$ levels rise with increasing muscle mass. It appears to be widely accepted that in children 'renal function can be estimated from serum creatinine'. ${ }^{4}$ Schwartz et al. ${ }^{5}$ and Counahan et al. ${ }^{6}$ each derived the hypothetical relationship between height $(\mathrm{Ht})$ and reciprocal $\mathbf{P}_{\mathbf{C}}$ and then confirmed this in studies on three series of patients comparing the estimated with measured GFR by creatinine and inulin clearances and CEDTA. Both groups found the relationship of predicted to measured GFR to be linear and concluded that individual $\mathbf{H t} / \mathbf{P}_{\mathbf{C}}$ estimates of GFR were valid.

We have repeated the comparison on 199 children aged between 3 and 16 years whose measured GFRs were distributed between severe impairment and normality. Our results do not accord with previous work.

\section{Method}

Between January 1977 and December 1978, CEDTA was performed on 107 boys and 92 girls aged between 3 and 16 years, who simultaneously had plasma creatinine and height measured. Because of the known variation in GFR proportional to surface area that occurs in early childhood ${ }^{3}$ children under age 3 years were excluded, as were those who were known to be morphometrically abnormal-for 
example those with spina bifida. The height was recorded in $\mathrm{cm}$ using a Harpenden stadiometer, and the weight in $\mathrm{kg}$. Surface area was calculated for each child from height and weight using the formulae of DuBois and DuBois ${ }^{7}$ and Haycock et al. ${ }^{8}$ Data were analysed using an ICL 1906A computer with software written for the statistical routines to conform with the methods of Armitage. ${ }^{9}$ The slope clearances were performed by single compartmental model analysis, and the result expressed as $\mathrm{ml} / \mathrm{min}$ per $1.73 \mathrm{~m}^{2}$ surface area. Two blood samples were taken, the first 2 hours after intravenous injection of isotope, and the second at 3,4 , or 6 hours, depending on the expected GFR. Levels of plasma creatinine were determined by a reaction rate method using a Union Carbide centrifugal analyser on a blood specimen drawn immediately before the injection of isotope, and the result expressed as $\mu \mathrm{mol} / \mathrm{l}$.

\section{Results}

The $\mathbf{H t} / \mathbf{P}_{\mathbf{C}}$ was plotted arithmetically against

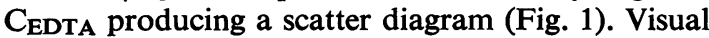
inspection suggests that the relationship was nonlinear over the full range. We therefore tested for linearity of the relationship using two methods.

(1) The data were tested in the form:

$\log \mathbf{C}_{\text {EDTA }}=\log \mathrm{a}+\mathrm{b} \log \mathrm{Ht}+\log \mathrm{P}_{\mathrm{C}}$ as reported previously by Counahan et al. $^{6}$ in their study of 108 children and adults. These authors found that the coefficients $b=-c=1$, and concluded that the relationship was linear. We were unable to confirm this but derived the values $b=0.91, c=1 \cdot 16$, the latter differing significantly from unity (Student's $t$ test for $\mathrm{b} ; t=0 \cdot 96$; for $\mathrm{c}, t=-5 \cdot 80$ ).

(2) Because in clinical practice the $\mathrm{Ht} / \mathbf{P}_{C}$ is used we examined the function:

$\mathrm{C}_{\text {EDTA }}=\mathbf{a} \times\left(\mathrm{Ht} / \mathrm{P}_{\mathrm{C}}\right)^{\mathrm{b}}$ in the form $\log \mathrm{C}_{\text {EDTA }}=\mathrm{Log}$ $a+b \log \left(H_{t} / P_{C}\right)$, and derived the following relationship:

$\log _{n} C_{\text {EDTA }}=3 \cdot 838+1 \cdot 151 \log _{n}\left(\mathrm{Ht} / \mathrm{P}_{\mathrm{C}}\right)$.

The standard error of $\mathrm{b}$ is 0.0272 and $t$ test for $\mathrm{b}=1, t=5.55$ which is significantly different from unity $(P<0.001)$. Retesting with $\log _{n} C_{\text {EDTA }}$ corrected for surface area using the Haycock, Schwartz, and Wisotsky formula generated the regression equation:

$\log _{n} C_{\text {EDTA }}=3 \cdot 834+1 \cdot 152 \log _{n}\left(\mathrm{Ht} / \mathrm{P}_{\mathrm{C}}\right)$.

The difference between the two surface area correction methods was so small that the following data analysis was based exclusively on Dubois.

The $95 \%$ confidence limits for individual values of $\mathrm{C}_{\text {EDTA }}$ from $\mathrm{Ht} / \mathrm{P}_{\mathrm{C}}$ were derived ${ }^{9}$ and these proved to be very wide (Fig. 2). Nevertheless the scatter diagram (Fig. 1) suggests that at low levels of GFR the relationship of $\mathrm{C}_{\mathrm{EDTA}}$ to $\mathrm{Ht} / \mathrm{P}_{\mathrm{C}}$ may be

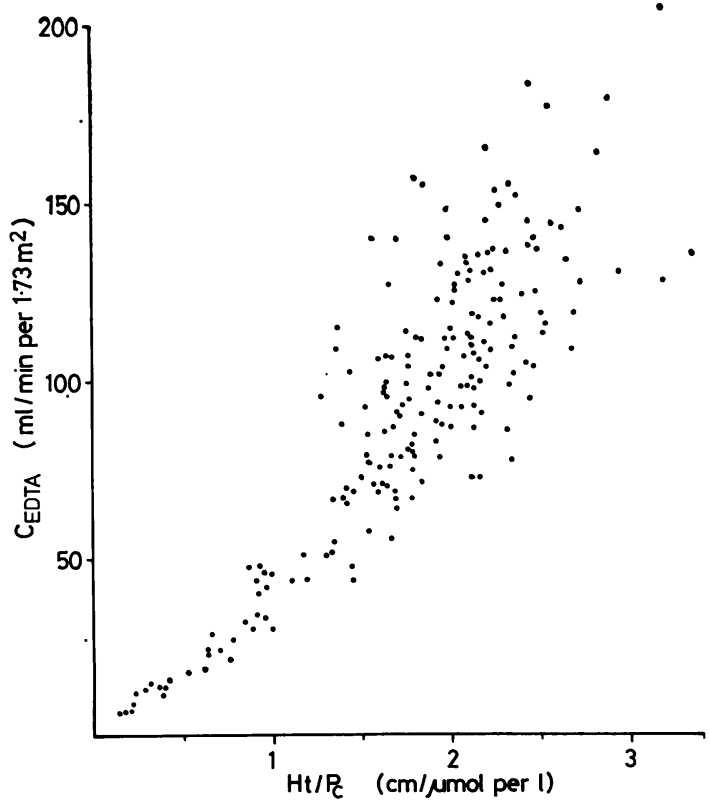

Fig. 1 Scatter diagram of $C_{E D T A}$ against $H t / P_{C}$ in 199 children.

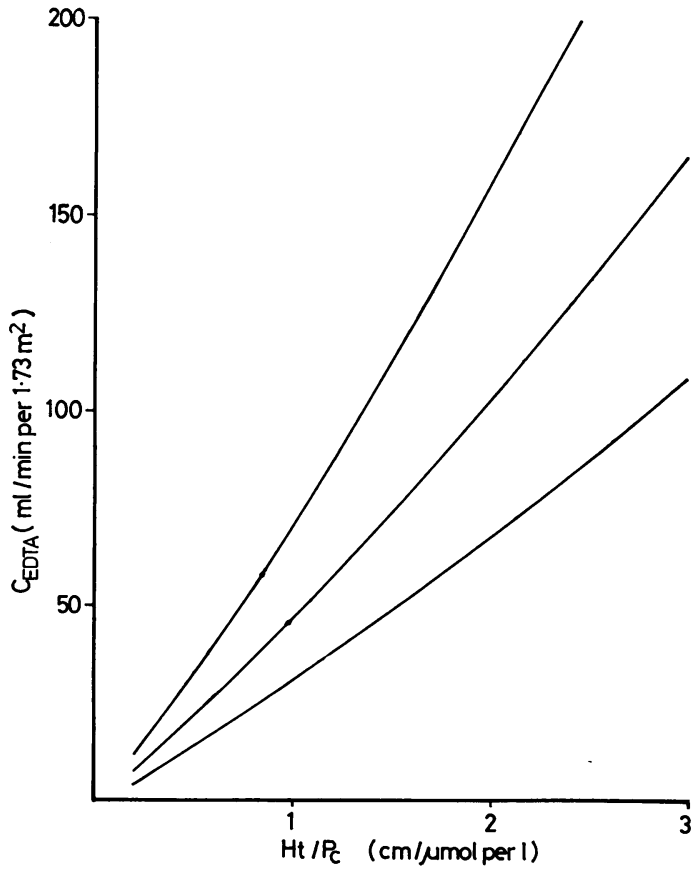

Fig. 2 Relationship between $C_{E D T A}$ and $\mathrm{Ht} / \mathrm{P}_{\mathrm{C}}$ showing $95 \%$ confidence limits. 
sufficiently linear and the residual variance among the observations sufficiently small to permit interpolation. Testing at successive incremental intervals of $\mathrm{Ht} / \mathbf{P}_{\mathrm{C}}$ of 0.2 indicated that at $\mathrm{Ht} / \mathbf{P}_{\mathrm{C}} \leqslant 1.2$ the

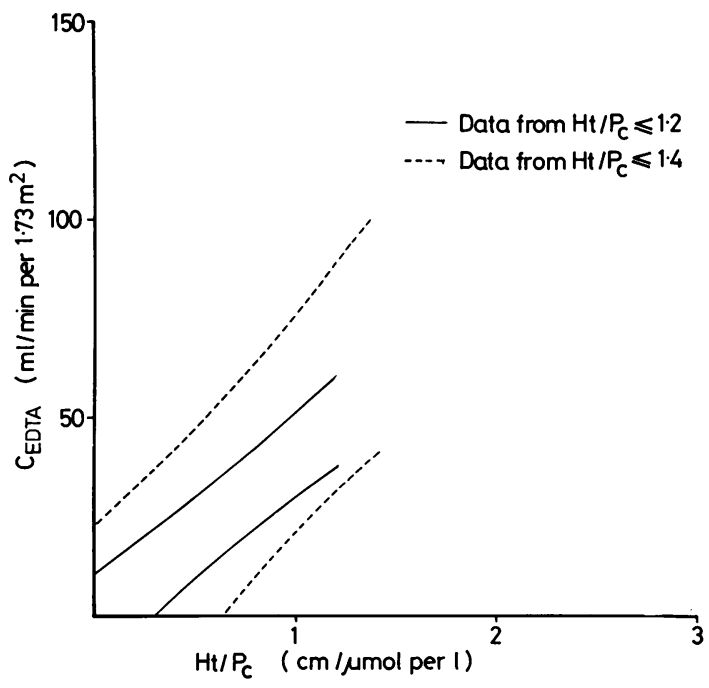

Fig. $395 \%$ confidence limits of $C_{E D T A}$ separately derived from individual patient data where $\mathrm{Ht} / \mathrm{P}_{\mathrm{C}}$ $\leqslant 1 \cdot 2$ and $\leqslant 1 \cdot 4$.

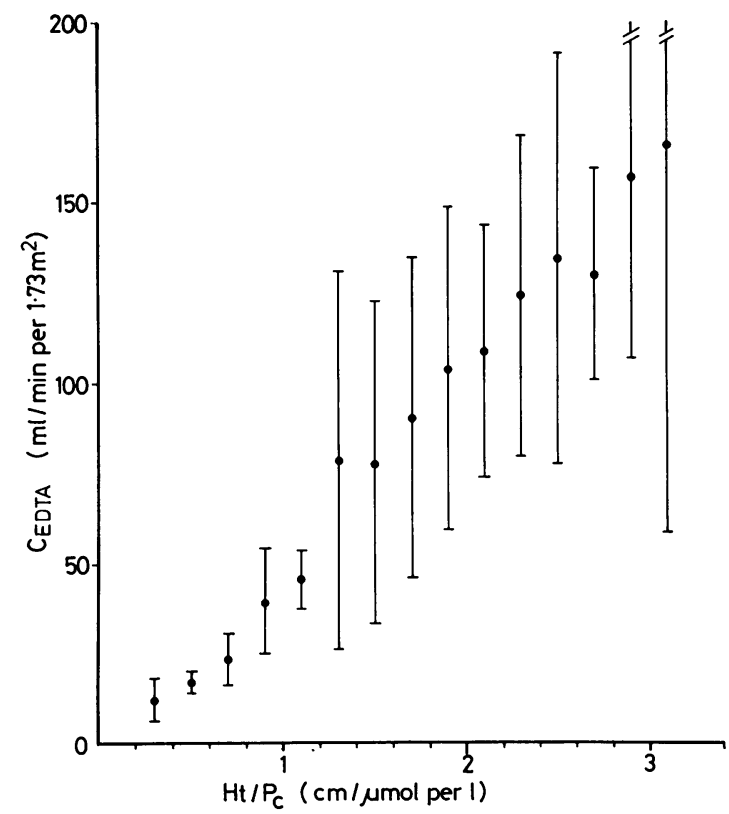

Fig. 4 Mean $\pm 2 S D$ for $C_{E D T A}$ at $0 \cdot 2$ intervals of $\mathrm{Ht} / \mathrm{PC}$. relationship could be reasonably represented linearly with the formula:

$$
\mathrm{C}_{\text {EDTA }}=-0 \cdot 78+41 \cdot 19\left(\mathrm{Ht} / \mathrm{P}_{\mathrm{C}}\right) \text {. }
$$

This is supported by logarithmic analysis as in (2) above, where the regression coefficient $b$ is not significantly different from 1 on this limited section of data $(b=0.97, \mathrm{SE}=0.049)$.

Inclusion of observations in the next interval to $\mathrm{Ht} / \mathrm{P}_{\mathrm{C}} \leqslant 1.4$ resulted in a certain loss of linearity in the logarithmic relationship $(b=1.096, S E$ of $b=0.059$ ) and widening of the confidence limits (Fig. 3). Above this level linearity was lost.

In Fig. 4, the mean \pm 2 standard deviations of $\mathrm{C}_{\mathrm{EDTA}}$ determinations has been located at the centre of successive $\mathrm{Ht} / \mathrm{P}_{\mathrm{C}}$ intervals of $\mathbf{0 . 2}$ where at least five observations were obtained. This graphically illustrates the abrupt dispersion of data at $\mathrm{Ht}_{\mathbf{t}} / \mathbf{P}_{\mathbf{C}}$ $>1 \cdot 2$.

\section{Discussion}

In the estimation of GFR in childhood from single $\mathrm{Ht} / \mathrm{P}_{\mathrm{C}}$ measurements our results do not confirm previously published work and our interpretation reflects this. The relationship between measured and predicted GFR is not linear, although it can be considered so in the small subset of patients with $\mathrm{Ht} / \mathbf{P}_{\mathrm{C}} \leqslant 1 \cdot 2$. Although a complex function itself would be no bar to the prediction of GFR, the $95 \%$ confidence limits are so wide as to render a single estimation highly inaccurate. For example, in an individual with an $\mathbf{H t} / \mathbf{P}_{\mathbf{C}}$ of 1.0 the estimated GFR would lie between 31 and $71 \mathrm{ml} / \mathrm{min}$ per $1.73 \mathrm{~m}^{2}$ with $95 \%$ probability (Fig. 2). This uncertainty is unacceptable in clinical practice. Even at $\mathbf{H t} / \mathbf{P}_{\mathbf{C}}$ of $\leqslant 1 \cdot 2$ where the dispersion of the data is clearly smaller (Fig. 4) and the function can be considered linear, the generated confidence limits, although narrower (Fig. 3), are still too wide to permit accurate prediction. It is within this range of reduced renal function that precise knowledge of GFR is clinically important. Furthermore, the diverging confidence limits at $\mathrm{Ht} / \mathbf{P}_{\mathbf{C}}>1.2$ preclude the use of $\mathrm{Ht} / \mathrm{P}_{\mathbf{C}}$ in screening for normal renal function. Using the lower confidence limit shown in Fig. 2 the $\mathrm{Ht} / \mathbf{P}_{\mathbf{C}}$ must exceed 2.16 in order to predict an individual GFR $>80 \mathrm{ml} / \mathrm{min}$ per $1.73 \mathrm{~m}^{2}$ with $97.5 \%$ confidence.

We recognise that the use of $\mathrm{C}_{\text {EDTA }}$ as the reference standard overestimates actual GFR at normal and high range. ${ }^{2}$ This is due to an inherent defect in the single compartmental model which ignores other exponential effects due to the equilibration of radiopharmaceutical between plasma and extracellular space. The non-linearity we have demonstrated between $\mathrm{C}_{\mathrm{EDTA}}$ and $\mathrm{Ht} / \mathrm{P}_{\mathrm{C}}$ is in accordance 
with this. However, we did not consider it justified to resort to inulin clearance as a reference standard with its additional inconvenience for patients and laboratory. The accuracy of $\mathrm{C}_{\mathrm{EDTA}}$ in practice is such that the comparison with $\mathrm{Ht} / \mathbf{P}_{\mathbf{C}}$ is a valid exercise.

We conclude that the prediction of GFR in children from single $\mathrm{Ht}$ and $\mathrm{P}_{\mathrm{C}}$ measurements is of limited value in clinical practice and should only be applied with caution. With impaired renal function where a precise determination of GFR is essential, the method is too inaccurate. As a screening test for normal renal function it detected 57 out of 131 children but erroneously recognised as normal 2 out of 68 with a measured GFR of less than $80 \mathrm{ml} / \mathrm{min}$ per $1.73 \mathrm{~m}^{2}$. We stress that these conclusions apply only to an individual estimate of GFR; our data do not permit evaluation of sequential measurements of $\mathrm{Ht} / \mathrm{P}_{\mathrm{C}}$ to monitor rates of change of renal function in a particular patient.

The chemical determinations were carried out in the Department of Clinical Chemistry, under the direction of the late Dr D N Raine, and data processing was performed by $\mathbf{R} \mathbf{J}$ Lancaster, computer officer, Department of Social Medicine, University of Birmingham.

\section{References}

1 Winterborn M H, Beetham R, White R H R. Comparison of plasma disappearance and standard clearance techniques for measuring glomerular filtration rate in children with and without vesico-ureteric reflux. Clin Nephrol 1977; 7: 262-70.

2 Chantler C, Barratt T M. Estimation of glomerular filtration rate from plasma clearance of ${ }^{51}$ chromiumedetic acid. Arch Dis Child 1972; 47: 613-7.

3 McCrory W M. Developmental nephrology. Cambridge, Mass: Harvard University Press, 1972: 95-108.

4 Anonymous. Microscopic haematuria in childhood. Lancet 1980 ; i: 859-60.

5 Schwartz G J, Haycock G B, Edelmann C M, Jr, Spitzer A. A simple estimate of glomerular filtration rate in children derived from body length and plasma creatinine. Pediatrics 1976; 58: 259-63.

6 Counahan R, Chantler C, Ghazali S, Kirkwood B, Rose F, Barratt T M. Estimation of glomerular filtration rate from plasma creatinine concentration in children. Arch Dis Child 1976; 51 : 875-8.

7 Du Bois D, Du Bois E F. Clinical calorimetry. X. A formula to estimate the approximate surface area if height and weight be known. Arch Internal Med 1916; 17: 863-71.

8 Haycock G B, Schwartz G J, Wisotsky D H. Geometric method for measuring body surface area: a heightweight formula validated in infants, children, and adults. J Pediatr 1978; 93: 62-6.

9 Armitage P. Statistical methods in medical research. Oxford: Blackwell, 1971: 164

Correspondence to Dr J G Davies, Department of Paediatrics, Walsgrave Hospital, Clifford Bridge Road, Walsgrave, Coventry, Warwickshire.

Received 20 April 1982

See commentary on page 614 . 